\section{Correlação e concordância entre indicadores de obesidade central e índice de massa corporal em adolescentes}

\section{Correlation and agreement between central obesity indicators and body mass index in adolescents}

Vera Lucia Chiara'

Henyse G.V. da Silva'

Maria Elisa Barros'

Ana Lúcia Rêgo'

Adriana Lúcia Ferreira"

Bruna A. Pitasi'

Thaís S. Mattos'

' Instituto de Nutrição da Universidade do Estado do Rio de Janeiro

"Faculdade de Ciências Médicas da Universidade do Estado do Rio de Janeiro

Fonte Financiadora: FAPERJ - E-26/150.142/2007 - bolsa de iniciação científica

Correspondência: Vera Lucia Chiara. Rua Deolinda Thurler, 36 Cônego. Nova Friburgo, RJ CEP 28 621 170. E-mail: chiara@uerj.br

\section{Resumo}

Objetivo: Analisar o desempenho de indicadores de obesidade central por correlação e concordância com índice de massa corporal para avaliação nutricional em adolescentes segundo sexo e idade. Métodos: Estudo seccional do tipo censo incluindo 635 adolescentes, de 10 a 19 anos (342 do sexo masculino e 293 do feminino), realizado em escola pública da cidade do Rio de Janeiro. Variáveis do estudo: índice de massa corporal (IMC), circunferências de cintura e abdômen, razões cintura-quadril e cintura/altura, estado nutricional, sexo e idade. Desenvolveram-se análise univariada e bivariada controlada por estratificação. Resultados: As circunferências de cintura e abdômen correlacionaram-se positiva e significativamente $(p<0,05)$ com o IMC em ambos os sexos, faixa etária e estado nutricional, enquanto a correlação de razão cintura/altura foi influenciada por sexo e estado nutricional. Para razão cintura/ quadril, a variação do sentido da correlação foi influenciada ainda por idade. As circunferências de cintura e abdômen foram dependentes da variação dos valores de IMC em todos os grupos com elevados $\mathrm{R}^{2}$ $(0,66$ a 0,80$)$ e $\beta$ positivo, o que não ocorreu para razões cintura/quadril e cintura/altura. Somente a circunferência de cintura apresentou agregamento moderado com o IMC (Índice Kappa de 0,41 a 0,57) em todos os estratos, enquanto nos demais indicadores o agregamento variou de relativo a moderado. Conclusões: Dentre os indicadores de obesidade central analisados, as circunferências de cintura e abdômen apresentaram os melhores desempenhos, destacando-se a circunferência de cintura como mais apropriada para uso complementar ao IMC na avaliação nutricional de adolescentes.

Palavras-chave: Adolescentes. Avaliação nutricional. Obesidade. Obesidade central. Indicadores nutricionais. 


\section{Abstract}

Objective: To assess the performance of central obesity indicators by correlation and agreement considering the body mass index in the nutritional assessment of adolescents according to sex and age. Methods: Cross-sectional census study with 635 adolescents, aged 10-19 years (342 males and 293 females), studying in a public school in Rio de Janeiro, Brazil. Study variables were body mass index (BMI), waist and abdominal circumference, waist-to-hip ratio, waist-to-height ratio, nutritional status, sex and age. Univariate and multivariate analyses adjusted by stratification were developed. Results: Waist and abdominal circumferences and BMI were positively and significantly correlated to sex, age group and nutritional status $(p<0.05)$. For the waist-to-hip ratio, co-relation variation was also influenced by age $(p<0.05)$. Waist and abdominal circumferences depended on BMI values in all groups $\left(\mathrm{R}^{2}=0.66-0.80\right.$, positive $\beta$ ), which did not occur in waistto-hip ratio and waist-to-height ratio. Only the waist circumference showed moderate agreement with BMI (Kappa: 0.41-0.57) in all groups, while for the remaining indicators this agreement ranged from poor to moderate. Conclusions: Among the central obesity indicators analyzed, waist and abdominal circumferences showed the best performances, but waist circumference was the most adequate to be used in addition to BMI in the nutritional assessment of adolescents.

Keywords: Adolescence. Nutritional assessment. Obesity. Central obesity. Nutritional indicators.

\section{Introdução}

Evidências crescentes demonstram que o excesso de gordura centralizada está associado à maior ocorrência de complicações metabólicas e doenças cardiovasculares em crianças, adolescentes e adultos ${ }^{1,2}$. A obesidade central tende a se elevar com o decorrer da idade, iniciando este processo ainda na fase de crescimento da infância e adolescência, e seguindo com o avançar da idade adulta. Existem diversas hipóteses quanto aos possíveis mecanismos biológicos sob os quais isto se processa ${ }^{3-5}$.

Os estudos de Taylor et al. ${ }^{3}$, Maffeis et al. ${ }^{4}$, Savva et al. ${ }^{7}$ e Li et al. ${ }^{8}$ apontaram que as circunferências de cintura e abdômen e as razões cintura/quadril e cintura/altura estão entre os mais empregados indicadores antropométricos de obesidade central. Kortelainen et al. ${ }^{6}$ sugerem que as circunferências de cintura e de abdômen demonstram a condição do acúmulo de gordura centralizada, enquanto para Savva et al. ${ }^{7} \mathrm{e}$ Li et al. ${ }^{8}$ as razões cintura/quadril e cintura/ altura têm servido como indicadores da forma com que a gordura centralizada se distribui nessa região.

As medidas de circunferências de cintura e abdômen e as razões cintura/quadril e cintura/altura apresentaram importante correlação com o índice de massa corporal nos estudos de Kosen et al. ${ }^{9}$ e Ardern et al. ${ }^{10}$ realizados com adultos, levando os autores a apontarem o índice de massa corporal (IMC) como excelente indicador de gordura corporal. O IMC é habitualmente empregado para avaliação nutricional de adultos ${ }^{9,10}$ e adolescentes ${ }^{11}$. Contudo, ainda não existe consenso quanto à utilidade dos indicadores de obesidade central para a fase da adolescência $^{5,12}$.

Este estudo analisa o desempenho dos indicadores circunferências de cintura e abdômen, razões cintura/quadril e cintura/ altura por meio de correlação e concordância com o índice de massa corporal para a avaliação nutricional de adolescentes segundo sexo e faixa de idade. 


\section{População e métodos}

Estudo de delineamento transversal com amostra extraída da primeira fase de amplo projeto de pesquisa longitudinal iniciado em 2007 em uma escola pública da cidade do Rio de Janeiro. Esta etapa preliminar do projeto incluiu um censo para estabelecer o perfil nutricional dos alunos matriculados, totalizando 1004 escolares (crianças e adolescentes). Deste total, 775 eram adolescentes na faixa etária de 10 a 19 anos, sendo que 140 (18,05\%) não participaram do estudo por (76) estarem cursando o pré-vestibular ou por opção própria (64). Para o presente trabalho, a população estudada integrou todos os adolescentes participantes do censo escolar, consistindo de 635 adolescentes, divididos em 342 do sexo masculino e 293 do feminino.

O projeto, em sua íntegra, foi aprovado na Comissão de Ética da Universidade do Estado do Rio de Janeiro, com protocolo de $\mathrm{n}^{\circ}$ 043.3.2006.

As variáveis do estudo foram: sexo, idade (anos), índice de massa corporal (IMC $\left.=\mathrm{kg} / \mathrm{m}^{2}\right)$, circunferência de cintura $(\mathrm{cm})$, circunferência de abdômen $(\mathrm{cm})$, razão cintura-quadril, razão cintura/altura e estado nutricional.

A idade foi estratificada por faixas etárias distintas entre os sexos, baseando-se na diferenciação do processo maturação sexual quando importantes mudanças nos compartimentos de massa gorda e magra ocorrem de forma diferenciada com a idade ${ }^{13}$.

A avaliação do estado nutricional foi feita por sexo e idade, utilizando-se o IMC, que é o indicador mais amplamente utilizado para avaliação de adolescentes. Os pontos de corte foram definidos para baixo peso, peso adequado, sobrepeso e obesidade. Para esta classificação nutricional adotaram-se as novas recomendações da Organização Mundial da Saúde ${ }^{14}$, recomendadas pelo Ministério da Saúde do Brasil ${ }^{15}$ em 2007. A condição de baixo peso situou-se abaixo do percentil 3 (exclusive), valores de IMC entre o percentil 3 (inclusive) e o percentil 85 (exclusive) para peso adequado, o sobre- peso a partir do percentil 85 (inclusive) até o percentil 97 (exclusive), e para obesidade os adolescentes com valores de IMC iguais ou superiores ao percentil $97^{15}$. Por vezes, para aplicação de testes estatísticos, os adolescentes foram agrupados em com excesso de peso (sobrepeso e obesidade) e sem excesso de peso (baixo peso e peso adequado).

As medidas de circunferências de cintura e abdômen, e as razões cintura/ quadril e cintura/altura foram escolhidas como indicadores nutricionais de gordura centralizada ${ }^{3,4,7,8}$.

As medidas antropométricas foram obtidas durante o horário da aula de educação física dos adolescentes, com os mesmos vestindo roupas leves próprias para as atividades e avaliados sem sapatos. Para obter as medidas de circunferências os alunos afastaram as vestimentas das áreas que foram aferidas. Todas as medidas corporais foram obtidas por antropometristas que incluíam mestrandas e bolsistas de pesquisa. A equipe foi treinada por um dos professores pesquisadores que elaborou manual para aferição de medidas antropométricas do projeto, visando obter precisão e exatidão, e adotando as técnicas de aferição estabelecidas por Lohman et al. ${ }^{16}$. Utilizou-se balança eletrônica SECA com capacidade para $150 \mathrm{~kg}$, estadiômetro fixado em parede sem rodapé e fitas métricas milimetradas não elásticas. Todas as medidas foram feitas em duplicatas, empregando-se a média aritmética como medida final.

\section{Análise dos dados}

O estudo incluiu análise descritiva das variáveis antropométricas, comparandose por sexo e faixa de idade por aplicação dos testes $t$ de Student (dados paramétricos: IMC, circunferências de cintura e de abdômen) e Mann-Whitney (dados não paramétricos: razões de cintura/quadril e cintura/altura). Determinou-se a prevalência do estado nutricional comparando por teste quiquadrado $\left({ }^{2}\right)$, com os adolescentes estratificados por sexo e grupos de com e sem excesso de peso. 
Aplicou-se análise bivariada integrando quatro testes para verificar, por meio de diferentes estratégias, o desempenho dos indicadores de obesidade central selecionados e definir aquele que apresentasse melhor correlação e concordância com o IMC para uso integrado na avaliação nutricional de adolescentes. Para tanto, empregou-se: gráfico de dispersão, correlação de Pearson, modelo de regressão linear e teste de concordância (índice Kappa).

Através dos gráficos de dispersão verificaram-se as tendências na distribuição e respectiva evolução dos valores de pares de indicadores de obesidade central em relação aos valores do IMC, por sexo e faixa de idade. A correlação de Pearson determinou a associação linear através do valor e significância do coeficiente $r$ como expressão do grau e sentido da direção nesta relação entre os indicadores de obesidade central e o IMC. A dependência entre os mesmos foi estabelecida através de modelo de regressão linear, adotando-se os indicadores de obesidade central (circunferências de cintura e abdômen, razões cintura/quadril e cintura/altura) como variáveis dependentes e o IMC como variável independente. Este resultado foi expresso através dos coeficientes de regressão $(\beta)$ e coeficiente de determinação ajustado $\left(\mathrm{R}^{2}\right)$. O valor de $\beta$ demonstrou a influência que cada unidade de IMC impôs sobre cada valor de qualquer dos indicadores de obesidade central analisados. $\mathrm{O} \mathrm{R}^{2}$ explicou quanto da variação do indicador de obesidade central pode ser predita (ou dependente) em razão da variação no IMC. Para ambos os testes considerou-se o nível de significância de $p$ $<0,05$, sendo os dados normalizados anteriormente por transformação em logaritmo (base 10). O grupo foi estratificado por sexo, faixa de idade e estado nutricional (sem e com excesso de peso).

Quanto à concordância considerou-se que, embora se estivesse trabalhando com métodos que aferem medidas corporais distintas, a análise de agregamento entre os indicadores de obesidade central e IMC contribuirá para definir qual dentre os mesmos poderia ter utilização simultânea com o IMC, contribuindo para avaliação nutricional mais detalhada. Desse modo, os dois indicadores seriam complementares e não permutáveis ${ }^{17}$. Para esta análise os adolescentes foram distribuídos por quartis de IMC, circunferências de cintura e abdômen, razões cintura/quadril e cintura/altura. A interpretação de agregamento para o valor do índice Kappa adotou o critério de Landis e Koch ${ }^{18}$, considerando-se: índice Kappa < $0=$ nenhum agregamento; índice Kappa de 0-0,19 = agregamento pobre; índice Kappa de 0,20-0,39 = agregamento relativo; índice Kappa de 0,40-0,59 = agregamento moderado; índice Kappa de 0,60-0,79 = agregamento substancial; e índice Kappa 0,80-1,00 = agregamento quase perfeito.

Os dados foram analisados com o programa SPSS for Windows, versão 11.0.1 ${ }^{19}$.

\section{Resultados}

As características do grupo revelaram que o sexo masculino apresentou valores significativamente mais elevados para os indicadores de obesidade central $(\mathrm{P}<0,05)$, não diferindo quanto ao IMC (Tabela 1). Embora predominasse a condição de peso adequado em todo o grupo, na comparação entre os estratos da faixa de mais idade o sexo masculino diferiu significativamente $(p$ $<0,01)$ do feminino quanto às prevalências das condições nutricionais. Exceção para o sexo feminino com idades $\geq 14$ anos apresentando sobrepeso, as demais prevalências de sobrepeso e obesidade foram sempre acima dos valores esperados (sobrepeso $=$ $12 \%$ e obesidade $=3 \%$ ), principalmente no sexo masculino (Tabela 2).

Através dos gráficos começaram a despontar as diferenças no desempenho dos indicadores de obesidade central, com os valores das circunferências de cintura e abdômen acompanhando de forma expressiva e positiva a distribuição e evolução do IMC em todos os estratos, o que não foi observado para as razões cintura/quadril e cintura/ altura (Figura 1).

Resultados similares para os sexos foram 
Tabela 1 - Estatística descritiva de índice de massa corporal (IMC) e circunferências de cintura e abdômen, razões cintura/quadril e cintura/altura dos adolescentes, por sexo e faixa etária*.

Table 1 - Descriptive statistics of body mass index (IMC), waist and abdominal circumferences, waist-to-hip and waist-toheight ratios of adolescents, by sex and age group*.

\begin{tabular}{|c|c|c|c|c|c|c|}
\hline Indicadores e Grupos & Média ( $\pm \mathrm{DP})$ & Mediana & $1^{\circ}$ Quartil & $3^{\circ}$ Quartil & Mínimo & Máximo \\
\hline \multicolumn{7}{|l|}{ Masculino } \\
\hline \multicolumn{7}{|l|}{$\mathrm{IMC}^{\mathrm{a}}$} \\
\hline$\leq 14$ anos $(n=183)$ & $20,86( \pm 3,63)$ & 20,50 & 17,99 & 23,00 & 13,49 & 34,74 \\
\hline$\geq 15$ anos $(n=159)$ & $22,75( \pm 3,57)$ & 22,02 & 20,29 & 24,93 & 16,64 & 35,09 \\
\hline \multicolumn{7}{|l|}{ C. cintura ${ }^{b}$} \\
\hline$\leq 14$ anos $(n=183)$ & $75,05( \pm 8,57)$ & 67,60 & 62,00 & 74,35 & 57,00 & 101,05 \\
\hline$\geq 15$ anos $(n=159)$ & $68,61( \pm 9,53)$ & 74,05 & 68,45 & 80,05 & 58,00 & 101,00 \\
\hline \multicolumn{7}{|l|}{ C. abdômen c } \\
\hline$\leq 14$ anos $(n=183)$ & $73,51( \pm 11,29)$ & 72,50 & 65,75 & 80,25 & 56,50 & 106,50 \\
\hline$\geq 15$ anos $(n=159)$ & $79,737( \pm 9,87)$ & 78,75 & 72,20 & 85,85 & 61,50 & 113,65 \\
\hline \multicolumn{7}{|l|}{ Razão cintura/quadril d } \\
\hline$\leq 14$ anos $(n=183)$ & $0,81( \pm 0,04)$ & 0,81 & 0,78 & 0,85 & 0,71 & 0,99 \\
\hline$\geq 15$ anos $(n=159)$ & $0,79( \pm 0,04)$ & 0,79 & 0,77 & 0,82 & 0,72 & 0,96 \\
\hline \multicolumn{7}{|l|}{ Razão cintura/altura e } \\
\hline$\leq 14$ anos $(n=183)$ & $0,44( \pm 0,05)$ & 0,43 & 0,40 & 0,48 & 0,32 & 0,70 \\
\hline$\geq 15$ anos $(n=159)$ & $0,43( \pm 0,04)$ & 0,42 & 0,40 & 0,47 & 0,32 & 0,58 \\
\hline \multicolumn{7}{|l|}{ Feminino } \\
\hline \multicolumn{7}{|l|}{ IMC a } \\
\hline$\leq 13$ anos $(n=125)$ & $20,05( \pm 4,01)$ & 19,12 & 17,45 & 21,74 & 13,01 & 34,88 \\
\hline$\geq 14$ anos $(n=168)$ & $21,96( \pm 3,68)$ & 21,37 & 19,51 & 23,19 & 16,00 & 35,14 \\
\hline \multicolumn{7}{|l|}{ C. cintura ${ }^{b}$} \\
\hline$\leq 13$ anos $(n=125)$ & $64,93( \pm 8,69)$ & 62,75 & 59,50 & 69,25 & 51,75 & 98,75 \\
\hline$\geq 14$ anos $(n=168)$ & $67,73( \pm 7,57)$ & 67,05 & 62,45 & 72,25 & 51,00 & 91,35 \\
\hline \multicolumn{7}{|l|}{ C. abdômen ${ }^{c}$} \\
\hline$\leq 13$ anos $(n=125)$ & $71,31( \pm 9,79)$ & 69,60 & 65,20 & 77,00 & 57,25 & 104,85 \\
\hline$\geq 14$ anos $(n=168)$ & $75,70( \pm 8,94)$ & 74,62 & 69,91 & 79,95 & 59,00 & 103,50 \\
\hline \multicolumn{7}{|l|}{ Razão cintura/quadril d } \\
\hline$\leq 13$ anos $(n=125)$ & $0,77( \pm 0,05)$ & 0,76 & 0,73 & 0,80 & 0,67 & 0,95 \\
\hline$\geq 14$ anos $(n=168)$ & $0.72( \pm 0,03)$ & 0,72 & 0,70 & 0,75 & 0,63 & 0,87 \\
\hline \multicolumn{7}{|l|}{ Razão cintura/alturae } \\
\hline$\leq 13$ anos $(n=125)$ & $0,42( \pm 0,05)$ & 0,41 & 0,39 & 0,45 & 0,29 & 0,64 \\
\hline$\geq 14$ anos $(n=168)$ & $0,49( \pm 0,04)$ & 0,41 & 0,39 & 0,45 & 0,31 & 0,56 \\
\hline
\end{tabular}

*comparação de faixa etária entre os sexos por teste $t$ (IMC, circunferências de cintura e abdomen) e Mann-Whitney (razões cintura/quadril e cintura/altura). *comparison of age groups between sexes by t test (BMI, waist and abdominal circumferences) and Mann-Whitney test (waist-to-hip and waist-to-height ratios).

alMC: $\leq 14$ e $\leq 13: t=1,81 ; P($ bilateral $)=0,07^{\text {ns }} ; \geq 14$ e $\geq 15: t=1,97 ; P($ bilateral $)=0,05^{\text {ns }}$

${ }^{a} B M I: \leq 14$ and $\leq 13: t=1.81 ; P($ bilateral $)=0.07^{n s} ; \geq 14$ and $\geq 15: t=1.97 ; P($ bilateral $)=0.05^{\text {ns }}$

${ }^{\mathrm{b} C}$.cintura: $\leq 14$ e $\leq 13: t=3,51 ; p($ bilateral $)<0,01^{\text {sig }} ; \geq 14$ e $\geq 15: t=8,16 ; p($ bilateral $)<0,01^{\text {sig }}$

${ }^{b}$ C.waist: $\leq 14$ and $\leq 13: t=3.51 ; p$ (bilateral) $<0.01^{\text {sig }} ; \geq 14$ and $\geq 15: t=8.16 ; p$ (bilateral) $<0.01^{\text {sig }}$

${ }^{c} C$. abdômen: $\leq 14$ e $\leq 13: t=1,81 ; p($ bilateral $)=0,07^{\text {ns; }} \geq 15$ e $\geq 14: t=3,86 ; p$ (bilateral $)<0,01^{\text {sig }}$

${ }^{c}$ C. abdominal: $\leq 14$ and $\leq 13: t=1.81 ; p$ (bilateral) $=0.07^{\text {ns }} ; \geq 15$ and $\geq 14: t=3.86 ; p$ (bilateral) $<0.01^{1^{\text {sig }}}$

dRazão cintura/quadril: $\leq 14$ e $\leq 13: U=6097,00 ; p<0,01^{\text {sig }} ; \geq 15$ e $\geq 14: U=2197,00 ; p<0,01^{\text {sig }}$

${ }^{d}$ waist-to-hip ratio: $\leq 14$ and $\leq 13: U=6097.00 ; p<0.01^{\text {sig }} ; \geq 15$ and $\geq 14: U=2197.00 ; p<0.01^{\text {sig }}$

eRazão cintura/altura: $\leq 14$ e $\leq 13: \mathrm{U}=9141,50 ; p<0,01^{\text {sig; }} \geq 15$ e $\geq 14: U=10978,00 ; p<0,01^{\text {sig }}$

${ }^{e}$ waist-to-height ratio: $\leq 14$ and $\leq 13: U=9141.50 ; p<0.01^{\text {sig }} ; \geq 15$ and $\geq 14: U=10978.00 ; p<0.01^{\text {sig }}$ 
Tabela 2 - Prevalência (\%) do estado nutricional dos adolescentes segundo índice de massa corporal, por sexo e faixa etária*.

Table 2 - Prevalence (\%) of nutritional status of adolescents according to body mass index, by sex and age group*.

\begin{tabular}{|c|c|c|c|c|c|}
\hline \multirow[t]{2}{*}{ Sexo/Idade } & \multirow[t]{2}{*}{$\mathbf{N}$} & \multicolumn{4}{|c|}{ Estado Nutricional (\%) } \\
\hline & & Baixo peso & Peso adequado & Sobrepeso & Obesidade \\
\hline \multicolumn{6}{|l|}{ Masculino } \\
\hline$\leq 14 \operatorname{anos}^{a}$ & 183 & 1,64 & 58,47 & 20,21 & 19,67 \\
\hline$\geq 15$ anos $^{b}$ & 159 & - & 54,09 & 27,04 & 18,86 \\
\hline \multicolumn{6}{|l|}{ Feminino } \\
\hline$\leq 13 \operatorname{anos}^{a}$ & 125 & 3,20 & 66,40 & 17,6 & 12,8 \\
\hline$\geq 14$ anos $^{b}$ & 168 & 1,19 & 80,35 & 11,31 & 7,14 \\
\hline
\end{tabular}

"comparação por faixa etária entre os sexos agrupados em sem e com excesso de peso.

"comparison by age range among sexes grouped into overweight and non-overweight.

${ }^{a} \leq 14$ anos e $\leq 13$ anos: $\chi^{2}=3,90$ e $p=0,27^{\text {ns; }}{ }^{a} \leq 14$ years and $\leq 13$ years: $\chi^{2}=3.90$ and $p=0.27^{\text {ns }}$

${ }^{b} \geq 15$ anos $e \geq 14$ anos: $\chi^{2}=29,64$ e $p<0,01^{\text {sig. }}{ }^{b} \geq 15$ years and $\geq 14$ years: $\chi^{2}=29.64$ and $p<0.01^{\text {sig. }}$.

observados na correlação positiva e significativa $(p<0,05)$ entre as circunferências de cintura e abdômen com o IMC em todos os estratos. O mesmo resultado foi observado com os modelos de regressão, embora o IMC influenciasse em algum grau e em diferentes sentidos as variações das razões cintura/quadril e cintura/altura entre os vários estratos (Tabela 3 ).
No conjunto dos indicadores de obesidade central, em todos os estratos as circunferências de cintura e abdômen foram os indicadores que demonstraram melhor agregamento com IMC, enquanto as razões cintura/quadril e cintura/altura revelaram flutuações no agregamento entre os estratos (Tabela 4).
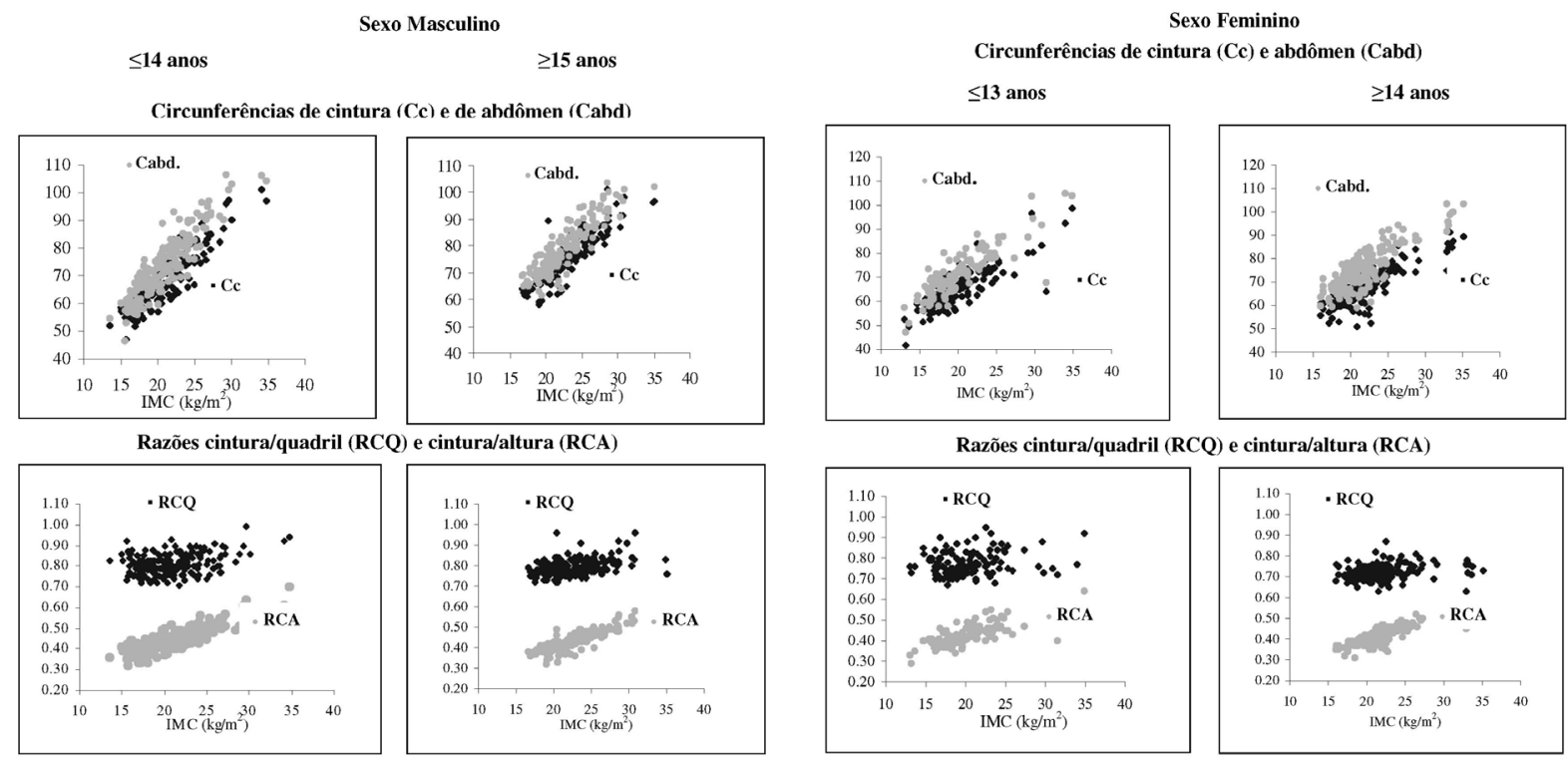

Figura 1 - Diagrama de dispersão para circunferências de cintura e abdômen, razões cintura/quadril e cintura/altura com índice de massa corporal (IMC) em adolescentes, por sexo e faixa de idade.

Figure 1 - Dispersion diagram for waist and abdominal circumferences, waist-to-hip and waist-to-height ratios with body mass index (IMC) of adolescents, by sex and age group. 
Tabela 3 - Coeficiente de correlação $(r)$ e valor de $p$, coeficientes de regressão $(\beta)$ e determinação ajustado $\left(R^{2}\right)$ para circunferências de cintura e abdômen e razões cintura/quadril e cintura/altura com índice de massa corporal segundo sexo, faixa de idade e estado nutricional.

Table 3 - Correlation coefficient $(r)$ and $p$ value, regression coefficients $(\beta)$ and adjusted determination $\left(R^{2}\right)$ for waist and abdominal circumferences, and waist-to-hip and waist-to-height ratios with body mass index by sex, age group and nutritional status.

\begin{tabular}{|c|c|c|c|c|c|c|c|c|c|c|c|c|}
\hline \multirow{2}{*}{$\begin{array}{l}\text { Sexo } \\
\text { F. idade } \\
\text { Est. Nut. }\end{array}$} & \multicolumn{3}{|c|}{ C. cintura } & \multicolumn{3}{|c|}{ C. abdomen } & \multicolumn{3}{|c|}{ Razão cintura/quadril } & \multicolumn{3}{|c|}{ Razão cintura/altura } \\
\hline & $r(p)$ & $\beta$ & $\mathrm{R}^{2}$ & $r(p)$ & $\beta$ & $\mathrm{R}^{2}$ & $r(p)$ & $\beta$ & $\mathrm{R}^{2}$ & $r(p)$ & $\beta$ & $\mathrm{R}^{2}$ \\
\hline \multicolumn{13}{|l|}{ Masc. } \\
\hline $\begin{array}{l}\leq 14 \text { Total } \\
(183)\end{array}$ & $\begin{array}{c}0,876 \\
\left(0,00^{*}\right)\end{array}$ & 0,705 & 0,73 & $\begin{array}{c}0,894 \\
\left(0,00^{*}\right)\end{array}$ & 0,802 & 0,80 & $\begin{array}{c}0,053 \\
\left(0,47^{\text {ns }}\right)\end{array}$ & 0,110 & 0,09 & $\begin{array}{c}0,811 \\
\left(0,00^{*}\right)\end{array}$ & 0,621 & 0,66 \\
\hline $\begin{array}{l}\text { Sem exc. } \\
\text { Peso } \\
(108)\end{array}$ & $\begin{array}{c}0,752 \\
\left(0,00^{*}\right)\end{array}$ & 0,506 & 0,36 & $\begin{array}{c}0,783 \\
\left(0,00^{*}\right)\end{array}$ & 0,481 & 0,34 & $\begin{array}{c}0,518 \\
\left(0,00^{*}\right)\end{array}$ & 0,051 & 0,04 & $\begin{array}{l}-0,150 \\
\left(0,11^{\text {ns }}\right)\end{array}$ & 0,577 & 0,48 \\
\hline $\begin{array}{l}\text { Exc. peso } \\
\text { (75) }\end{array}$ & $\begin{array}{c}0,857 \\
\left(0,00^{*}\right)\end{array}$ & 0,408 & 0,53 & $\begin{array}{c}0,872 \\
\left(0,00^{*}\right)\end{array}$ & 0,492 & 0,58 & $\begin{array}{c}0,768 \\
\left(0,00^{*}\right) \\
\end{array}$ & $-0,083$ & 0,08 & $\begin{array}{c}0,363 \\
\left(0,00^{*}\right) \\
\end{array}$ & 0,430 & 0,54 \\
\hline \multicolumn{13}{|l|}{$\geq 15$} \\
\hline $\begin{array}{l}\text { Total } \\
(159) \\
\end{array}$ & $\begin{array}{c}0,880 \\
\left(0,00^{*}\right) \\
\end{array}$ & 0,651 & 0,77 & $\begin{array}{c}0,887 \\
\left(0,00^{*}\right)\end{array}$ & 0,708 & 0,79 & $\begin{array}{l}-0.274 \\
\left(0,00^{*}\right)\end{array}$ & 0,136 & 0,16 & $\begin{array}{c}0,854 \\
\left(0,00^{*}\right) \\
\end{array}$ & 0,632 & 0,73 \\
\hline $\begin{array}{l}\text { S/ exc. } \\
\text { peso } \\
(86) \\
\end{array}$ & $\begin{array}{c}0,579 \\
\left(0,00^{*}\right)\end{array}$ & 0,563 & 0,34 & $\begin{array}{c}0,575 \\
\left(0,00^{*}\right)\end{array}$ & 0,521 & 0,32 & $\begin{array}{c}0,232 \\
\left(0,03^{*}\right)\end{array}$ & 0,453 & 0,22 & $\begin{array}{c}0,483 \\
\left(0,00^{*}\right)\end{array}$ & 0,145 & 0,04 \\
\hline $\begin{array}{l}\text { Exc. peso } \\
\text { (73) }\end{array}$ & $\begin{array}{c}0,812 \\
\left(0,00^{*}\right) \\
\end{array}$ & 0,629 & 0,65 & $\begin{array}{c}0,795 \\
\left(0,00^{*}\right) \\
\end{array}$ & 0,672 & 0,63 & $\begin{array}{c}0,334 \\
\left(0,00^{*}\right)\end{array}$ & 0,160 & 0,09 & $\begin{array}{c}0,819 \\
\left(0,00^{*}\right) \\
\end{array}$ & 0,698 & 0,65 \\
\hline \multicolumn{13}{|l|}{ Fem. } \\
\hline $\begin{array}{l}\leq 13 \\
\text { Total } \\
(125) \\
\end{array}$ & $\begin{array}{c}0,839 \\
\left(0,00^{*}\right)\end{array}$ & 0,576 & 0,70 & $\begin{array}{c}0,858 \\
\left(0,00^{*}\right)\end{array}$ & 0,61 & 0,73 & $\begin{array}{c}0,228 \\
\left(0,01^{*}\right)\end{array}$ & 0,08 & 0,04 & $\begin{array}{c}0,759 \\
\left(0,00^{*}\right)\end{array}$ & 0,50 & 0,58 \\
\hline $\begin{array}{l}\text { S/ exc. } \\
\text { peso } \\
(87) \\
\end{array}$ & $\begin{array}{c}0,705 \\
\left(0,00^{*}\right)\end{array}$ & 0,569 & 0,50 & $\begin{array}{c}0,731 \\
\left(0,00^{*}\right)\end{array}$ & 0,648 & 0,54 & $\begin{array}{l}-0,158 \\
\left(0,14^{\text {ns }}\right)\end{array}$ & $-0,086$ & 0,01 & $\begin{array}{c}0,562 \\
\left(0,00^{*}\right)\end{array}$ & 0,438 & 0,32 \\
\hline $\begin{array}{l}\text { Exc. Peso } \\
(38)\end{array}$ & $\begin{array}{c}0,672 \\
\left(0,00^{*}\right) \\
\end{array}$ & 0,546 & 0,40 & $\begin{array}{c}0,716 \\
\left(0,00^{*}\right) \\
\end{array}$ & 0,554 & 0,46 & $\begin{array}{c}-0,101 \\
\left(0,54^{\text {ns }}\right) \\
\end{array}$ & $-0,075$ & 0,00 & $\begin{array}{c}0,450 \\
\left(0,00^{*}\right) \\
\end{array}$ & 0,305 & 0,14 \\
\hline \multicolumn{13}{|l|}{$\geq 14$} \\
\hline $\begin{array}{l}\text { Total } \\
(168) \\
\end{array}$ & $\begin{array}{c}0,829 \\
\left(0,00^{*}\right) \\
\end{array}$ & 0,570 & 0,66 & $\begin{array}{c}0,843 \\
\left(0,00^{*}\right) \\
\end{array}$ & 0,605 & 0,68 & $\begin{array}{c}0,221 \\
\left(0,00^{*}\right) \\
\end{array}$ & 0,072 & 0,04 & $\begin{array}{c}0,857 \\
\left(0,00^{*}\right) \\
\end{array}$ & 0,592 & 0,72 \\
\hline $\begin{array}{l}\text { S/ exc. } \\
\text { peso } \\
(137) \\
\end{array}$ & $\begin{array}{c}0,611 \\
\left(0,00^{*}\right)\end{array}$ & 0,50 & 0,36 & $\begin{array}{c}0,593 \\
\left(0,00^{*}\right)\end{array}$ & 0,480 & 0,34 & $\begin{array}{c}0,093 \\
\left(0,27^{\mathrm{ns}}\right)\end{array}$ & 0,050 & 0,00 & $\begin{array}{c}0,704 \\
\left(0,00^{*}\right)\end{array}$ & 0,577 & 0,48 \\
\hline $\begin{array}{l}\text { Exc. Peso } \\
\text { (31) }\end{array}$ & $\begin{array}{c}0,747 \\
\left(0,00^{*}\right)\end{array}$ & 0,532 & 0,40 & $\begin{array}{c}0,789 \\
\left(0,00^{*}\right)\end{array}$ & 0,533 & 0,46 & $\begin{array}{c}-0,202 \\
\left(0,27^{\text {ns }}\right)\end{array}$ & $-0,072$ & 0,00 & $\begin{array}{c}0,750 \\
\left(0,00^{*}\right)\end{array}$ & 0,294 & 0,14 \\
\hline
\end{tabular}

*significativo $(p<0,05)$; ns não significativo

${ }^{*}$ significant $(p<0.05) ;{ }^{n s}$ not significant

\section{Discussão}

No presente estudo, verificou-se que os indicadores circunferências de cintura e abdômen apresentaram bom desempenho frente às razões cintura/quadril e cintura/ altura. No entanto, a circunferência de cintura destacou-se na correlação e concordância com o IMC em ambos os sexos, faixa de idade e estado nutricional, sendo 
Tabela 4 - Índice Kappa e valor de P para concordância entre as distribuições dos adolescentes por quartis de índice de massa corporal com circunferências de cintura e abdômen, razões cintura/quadril e cintura/altura, segundo sexo e faixa etária.

Table 4 - Kappa index and $P$ value for agreement between distributions of adolescents by quartiles of body mass index with waist and abdominal circumferences, waist-to-hip and waist-to-height ratios, by sex and age group.

\begin{tabular}{|c|c|c|c|c|c|c|c|c|c|}
\hline \multirow{3}{*}{$\begin{array}{l}\text { Sexo e faixa } \\
\text { etária }\end{array}$} & \multirow[t]{3}{*}{$\mathrm{N}$} & \multicolumn{2}{|c|}{ C. cintura } & \multicolumn{2}{|c|}{ C. abdomen } & \multicolumn{2}{|c|}{ Razão cintura/quadril } & \multicolumn{2}{|c|}{ Razão cintura/altura } \\
\hline & & Índice & $p$ & Índice & $p$ & Índice & $p$ & Índice & $p$ \\
\hline & & & & & & & & & \\
\hline \multicolumn{10}{|l|}{ Masculino } \\
\hline$\leq 14$ anos & 183 & $0,51^{c}$ & 0,000 & $0,56^{c}$ & 0,000 & $0,11^{\mathrm{a}}$ & 0,009 & $0,35^{b}$ & 0,000 \\
\hline$\geq 15$ anos & 159 & $0,55^{c}$ & 0,000 & $0,57^{c}$ & 0,000 & $0,09^{a}$ & 0,038 & $0,53^{c}$ & 0,000 \\
\hline \multicolumn{10}{|l|}{ Feminino } \\
\hline$\leq 13$ anos & 125 & $0,43^{c}$ & 0,000 & $0,52^{c}$ & 0,001 & $0,05^{a}$ & 0,324 & $0,28^{b}$ & 0,000 \\
\hline$\geq 14$ anos & 168 & $0,43^{c}$ & 0,000 & $0,36^{b}$ & 0,000 & $0,08^{\mathrm{a}}$ & 0,048 & $0,43^{c}$ & 0,000 \\
\hline
\end{tabular}

a Índice Kappa de 0 a 0,19= agregamento pobre; ${ }^{\mathrm{a}}$ Kappa Índex of 0 to $0.19=$ poor agreement

${ }^{\mathrm{b}}$ Índice Kappa de 0,20-0,39 = agregamento relativo; ${ }^{\mathrm{b}} \mathrm{Kappa}$ Index of 0.20-0.39= relative agreement

c Índice Kappa de 0,40-0,59= agregamento moderado; ${ }^{c}$ Kappa Índex of 0.40-0.59= moderate agreement

considerado o indicador de obesidade central a ser utilizado junto ao IMC na avaliação nutricional de adolescentes.

Diversos estudos empregando indicadores nutricionais, dentre os quais a circunferência de cintura, apontaram resultados similares. Assim, Lee et al. ${ }^{20}$ observaram mais expressiva correlação entre circunferência de cintura e IMC, quando comparada às razões cintura/quadril e cintura/altura em adolescentes. Para os autores, o sexo foi a variável que mais influenciou nesta relação, com o sexo masculino apresentando elevados valores de circunferência de cintura.

O estudo atual também encontrou no sexo masculino os mais altos valores de gordura centralizada com todos os indicadores utilizados, além de maior prevalência de excesso de peso. Este resultado está em acordo com a afirmativa de Moreno et al. ${ }^{21}$, que analisaram a distribuição de gordura em adolescentes obesos e não obesos, concluindo que a obesidade total vem sempre acompanhada do aumento nos depósitos de gordura central em diferentes regiões.

Em nosso estudo, os indicadores razões cintura/quadril e cintura/altura não apresentaram melhor desempenho do que as circunferências frente ao IMC, mesmo considerando-se a estratificação do grupo por sem e com excesso de peso corporal.
Consoante com estes resultados, a circunferência de cintura foi apontada por Neovius et $\mathrm{Al}^{22}$ como indicador de alta sensibilidade e especificidade em adolescentes, comparada à razão cintura/quadril e tomando como referência o percentual de gordura definido por pletismografia. Da mesma forma, o estudo de Taylor et al. ${ }^{3}$ considerou este indicador de obesidade central como o mais apropriado para adolescentes quando comparado à razão cintura/quadril. Estes autores tomaram por referência de gordura central o percentual de gordura obtido por absorciometria de duplo feixe de energia (DXA). A mesma afirmativa foi considerada na conclusão do estudo de Semiz et al. ${ }^{23}$.

Para Freedman et al. ${ }^{24}$, a importância da circunferência de cintura como indicador de gordura central em adolescentes se destacou nos resultados de estudo analisando a relação entre circunferências de cintura, de quadril, razão cintura/quadril, dobras subescapular e triciptal com níveis de lipídios séricos e concentração de insulina.

O trabalho de Li et al. ${ }^{8}$ demonstrou que. embora se observasse significativa correlação e concordância entre circunferência de cintura e razão cintura/altura, e ambos os indicadores tivessem captado o aumento nos teores de gordura central em adolescentes americanos no período de 1999 a 
2004, a circunferência de cintura sobressaiu como indicador de obesidade central em adolescentes.

No presente estudo, a circunferência de abdômen também apresentou desempenho junto ao IMC superior às razões cintura/ quadril e cintura/altura. No entanto, ainda são escassos os trabalhos com adolescentes que, de fato, empregaram este indicador. Segundo Lohman et al $^{16}$, a principal dificuldade com referência a esta questão está relacionada às limitações metodológicas dos estudos quanto à aferição das próprias medidas de circunferências, por vezes aferindo o local de um tipo de circunferência e no texto referindo-se à outra.

Neste contexto, detendo-se na análise de concordância observou-se que, em todos os estratos, a circunferência de cintura apresentou o melhor agregamento com maiores valores do índice Kappa, especialmente em adolescentes do sexo masculino, onde também prevaleceu o excesso de peso. Esta questão parece apontar para a observação de Bland \& Altman ${ }^{25}$ quanto a uso deste índice na análise de concordância. Para os estudiosos, o índice Kappa pode ser afetado pela característica de interesse no estudo que, no caso, seria considerado o excesso de peso.

Segundo Freitas e Pereira ${ }^{26}$, essa característica do índice Kappa contribui para considerar que o coeficiente pode não ser a medida mais adequada para avaliar o grau de concordância em estudos epidemiológicos de confiabilidade. No entanto, no estudo atual, a aplicação do teste de concordância não apresentou esta finalidade, considerando-se que os métodos de avaliação nutricional seriam complementares e não permutáveis.

Tendo em vista a finalidade do estudo atual quanto à análise do indicador de obesidade central para uso integrado ao IMC, ambos os indicadores de circunferência correlacionaram-se positiva e significativamente com o IMC em sexo, faixa etária e estado nutricional. Todavia, como Bland \& Altman ${ }^{27}$ alertaram que a correlação pode ser afetada com a variabilidade interindividual da medida, foram desenvolvidos modelos de regressão que confirmaram os resultados.

No presente estudo, atentou-se para controle de possíveis variáveis de confusão estratificando o grupo por sexo, faixa de idade e estado nutricional. No entanto, algumas limitações devem ser consideradas:

- divisão por faixa etária da população, entendendo-se que o mais indicado seja a utilização de estágios de maturação sexual. Entretanto, em face da dificuldade de obtenção dessa informação, o corte diferenciado nas idades procurou atender a questão da diferenciação da composição corporal frente o processo de crescimento e desenvolvimento no qual se insere a maturação sexual ${ }^{13}$;

- uso do IMC para orientar a seleção do indicador de gordura centralizada, pois, embora o mesmo seja considerado bom indicador de obesidade total, o mais apropriado seria adotar métodos laboratoriais mais precisos e acurados para diagnosticar gordura intra-abdominal;

- incerteza se os achados atuais podem ser generalizados, mesmo considerando que os dados se originam de pesquisa base do tipo censo que avaliou $82 \%$ da população.

A partir dos resultados apresentados, concluiu-se que, embora o desempenho das circunferências tenha sido similar, a circunferência de cintura destacou-se como o mais apropriado indicador de obesidade central em adolescentes para uso complementar ao IMC. Todavia, há necessidade de comprovações científicas mais aprofundadas e maior consenso entre os estudos para considerar esse achado.

Potencial Conflito de Interesse: Declaramos não haver conflito de interesse. 


\section{Referências}

1. Hirschler V, Aranda C, Calcagno Mde L, Maccalini G, Jadzinsky M. Can waist circumference identify children with the metabolic syndrome? Arch Pediatr Adolesc Med 2005; 159(8): 740-4.

2. Daniels SR, Khourty PR, Morrison JA. Utility of different measures of body fat distribution in children and adolescents. Am J Epidemiol 2000; 152(12): 1179-84.

3. Taylor RW, Jones IA, Williams SM, Goulding A. Evaluation of waist circumference, waist-to-hip ratio, and the conicity index as screening tools for high trunk fat mass, as measured by dual-energy X-ray absorptiometry, in children aged 3-19. Amer Jour Clin Nutr 2000; 72 (2): 490-5.

4. Maffeis C, Pietrobelli A, Grezzani A, Provera S, Tato L. Waist circumference and cardiovascular risk factors in prepubertal children. Obes Res 2001; 9 (3): 179-87.

5. Kuk JL; Lee S; Heymsfield SB; Ross R. Waist circumference and abdominal adipose tissue distribution: influence of age and sex. Amer J Clin Nutr 2005; 81(6): 1330-34.

6. Kortelainen ML, Särkioja T. Visceral fat and coronary pathology in male adolescents. Int J Obes Relat Metab Disord 2001; 25(2): 228-32.

7. Savva SC, Tornaritis M, Savva ME, Kourides Y, Silikiotou $\mathrm{N}$, Georgiou C et al. Waist circumference and waistto-height ratio are better predictors of cardiovascular disease risk factors in children than body mass index. Int J Obes Relat Metab Disord 2000; 24(11): 1453-8.

8. Li C, Ford ES, Mokdad AH, Cook S. Recent Trends in Waist Circumference and Waist-Height Ratio Among US Children and Adolescents. Pediatrics 2006; 118 (5): 1390-8.

9. Okosen IS; Tedders SH; Choi S; Dever GE. Abdominal adiposity values associated with established body mass indexes in white, black and Hispanic Americans. A study from the Third National Health and Nutrition Examination Survey. Int J Obes Relat Metab Disord 2000; 24(10): 1279-85.

10. Ardern C, Janssen I, Ross R, Katzmarzyk PT. Development of Health-Related Waist Circumference Thresholds Within BMI Categories. Obesity Research 2004; 12(7): 1094-103.

11. Rodrigues CA Prevalência de Excesso de Peso em Adolescentes Residentes na Zona Urbana de Porto Alegre [dissertação de mestrado]. Porto Alegre: Universidade Federal do Rio Grande do Sul. Faculdade de Medicina; 2006.

12. Almeida CAN, Pinho AP, Ricco RG, Elias CP. Abdominal circumference as an indicator of clinical and laboratory parameters associated with obesity in children and adolescents: comparison between two reference tables. $J$ Pediatr 2007; 83(2): 181-5.

13. Barros ME. Composição corporal de adolescentes de bom nível socioeconômico: determinação pelo método de absorção de duplo feixe de energia (DEXA) [dissertação de mestrado]. São Paulo: Escola Paulista de Medicina da Universidade Federal de São Paulo; 1999.
14. WHO. World Health Organization. Growth reference data for 5-19 years. Disponível em http://www.who.int/ growthref/en/2007 [Acessado em 1 de outubro de 2007].

15. Ministério da Saúde. Secretaria de Atenção à Saúde. Departamento de Atenção Básica. Coordenação-Geral da Política de Alimentação e Nutrição. Incorporação da curvas de crescimento da Organização Mundial da Saúde de 2006 e 2007 no SISVAN. Disponível em http: / / www. saude.gov.br/nutrição [Acessado em 10 de fevereiro de 2008].

16. Lohman TG, Roche AF, Martorell R. Anthropometric standardization reference manual. Illinois: Human Kinetics Books; 1988.

17. Bland JM, Altman DG. Statistical Methods For Assessing Agreement Between Two Methods of Clinical Measurement. The Lancet 1986; 11: 307-10.

18. Landis JR, Koch GG. The measurement of observer agreement for categorical data. Biometrics 1977; 33: 15974 .

19. SPSS. Statistical Package for the Social Sciences; 2001.

20. Lee S, Kuk JL, Hannon TS, Arslanian SA. Race and gender differences in the relationships between anthropometrics and abdominal fat in youth. Obesity 2008; 16(5): 1066-71.

21. Moreno LA; Fleta J; Mur L; Sarría A; Bueno M. Fat distribution in obese and nonobese children and adolescents. J Pediatr Gastroenterol Nutr 1998; 27(2): 176-80.

22. Neovius M, LinnéY, Rossner S. BMI, waist-circumference and waist-hip-ratio as diagnostic tests for fatness in adolescents. Inter J Obes 2005; 29(2): 163-9.

23. Semiz S; Ozgören E; Sabir N. Comparison of ultrasonographic and anthropometric methods to assess body fat in childhood obesity. Int J Obes 2007; 31(1): 538.

24. Freedman DS, Serdula MK, Srinivasan SR, and Berenson GS. Relation of circumferences and skinfold thicknesses to lipid and insulin concentrations in children and adolescents: the Bogalusa Heart Study. Amer J Clin Nutr 1999; 69(2): 308-17.

25. Bland JM; Altman DG. Applying the right statistics: analyses of measurement studies. Ultrasound Obstet Gynecol 2003; 22: 85-93.

26. Freitas ES, Pereira MG. Avaliação das estruturas de concordância e discordância nos estudos de confiabilidade. Rev Saúde Pública 1998; 32(4): 383-93.

27. Bland JM, Altman DG. Correlation, regression, and repeated data. BMJ 1994; 308: 896.

Recebido em: 09/10/08

Versão final reapresentada em: 01/06/09 Aprovado em: 23/06/09 